\title{
Hipertrigliceridemia Familiar Grave durante a Gestação
}

\author{
Severe Familial Hypertriglyceridemia during Pregnancy
}

Luiz Augusto Casulari, Myrian Wesgueber, Ricardo Cassiano B. Silva

Henrique F. Soares, Lucilia Domingues

\begin{abstract}
RESUM0
A presença de hipertrigliceridemia grave durante a gravidez é rara, mas comporta grande possibilidade de desenvolver complicações, como a pancreatite aguda, que coloca em risco a mãe e o feto. Apresentamos o relato da evolução da gestação de uma paciente portadora de hipertrigliceridemia grave que havia apresentado pancreatite aguda dois meses antes da fecundação. Foi tratada durante o pré-natal com dieta e 3,0 g de ácidos graxos de cadeia ômega-3 (ácidos eicosapentaenóico 14\% e docosahexaenóico 11,13\%). Os niveis de triglicerideos foram mantidos abaixo de $800 \mathrm{mg} / \mathrm{dL}$, sendo este limite considerado seguro para evitar o desenvolvimento de pancreatite aguda. A gestação evoluiu, sem intercorrências, para parto vaginal, a termo. O recém-nato não apresentou alterações morfológicas ao nascimento. Concluimos que, nesta paciente grávida e portadora de hipertrigliceridemia grave, uma dieta adequada e o emprego de ácidos graxos de cadeia ômega-3 foram eficazes em prevenir a pancreatite aguda. Esta abordagem terapêutica pode ser uma alternativa para as gestantes portadoras de hipertrigliceridemia familiar.
\end{abstract}

PALAVRAS-CHAVE: Hipertrigliceridemia. Lipoproteínas. Complicações da Gravidez. Pancreatite.

\section{Introdução}

Durante a gestação, mulheres normais apresentam um aumento dos niveis de triglicerídeos plasmáticos, ocorrendo o seu pico máximo na metade do terceiro trimestre, chegando a valores até cinco vezes maiores do que os valores basais ${ }^{1}$. Isso se deve ao aumento dos niveis de estrogênio e prolactina, que acarretariam maior produção hepática de lipoproteínas ricas em triglicerídeos (VLDL) e ao efeito lipolítico do hormônio lactogênico placentário sobre o tecido $\operatorname{adiposo}^{1,2}$.

Já as portadoras de hipertrigliceridemia familiar apresentam, durante a gestação, piora importante nos seus niveis sangüíneos de triglicerideos e quilomicrons, ocasionando um ris-

Hospital Prontonorte, Brasília - DF.

Correspondência: Luiz Augusto Casulari

SHIN QL 2 conjunto 12 casa 2 - Lago Norte

71510-125 - Brasília - DF

e-mail: roxomotta@ambr.com.br co potencial de desenvolvimento de pancreatite aguda e suas complicações, tais como: hemorragia intraperitoneal e gastrointestinal, trombose de veia esplênica, obstrução e necrose intestinal, septicemia e choque ${ }^{3,4}$. Nessas condições, a mortalidade materna é estimada em $21 \%$ e a do feto em $20 \%{ }^{5}$.

Com o objetivo de prevenir essas indesejáveis complicações, várias estratégias têm sido preconizadas para diminuir os altos níveis de triglicerideos em pacientes portadoras de hipertrigliceridemia familiar, como: nutrição parenteral total ${ }^{6}$, plasmaferese e heparinização ${ }^{4}$, associadas à dieta. No entanto, esses tratamentos podem apresentar efeitos colaterais e colocar em risco a vida da paciente.

Neste artigo, apresentamos o relato de uma paciente que foi tratada da hipertrigliceridemia grave com dieta e ácidos graxos de cadeia ômega3 e que teve boa evolução na sua gravidez. 


\section{Relato do Caso}

Paciente com 25 anos, branca, foi atendida pela primeira vez com quadro de dor abdominal e vômitos, dois meses antes da gravidez. Apresentava pressão arterial sistêmica de $120 \times 80 \mathrm{mmHg}$, altura de $154 \mathrm{~cm}$, peso de $52 \mathrm{~kg}$ e índice de massa corporal (IMC) de $21,9 \mathrm{~kg} / \mathrm{m}^{2}$. Não era portadora de xantomatose. Na ocasião possuía níveis séricos de amilase $=150 \mathrm{U} / \mathrm{L}(\mathrm{VN}: 25$ a $125 \mathrm{U} / \mathrm{L})$, lipase $=$ 20 U/1 (VN: 2 a 18 U/L), cálcio = 10,4 mg/dL (VN: 8 a $11 \mathrm{mg} / \mathrm{dL})$, glicose $=235 \mathrm{mg} / \mathrm{dL}(\mathrm{VN}$ : 70 a 110 $\mathrm{mg} / \mathrm{dL}$ ) e leucócitos $=11.700 / \mathrm{mm}^{3}$ (VN: $3600 \mathrm{a}$ $11000 / \mathrm{mm}^{3}$ ) A ecografia e a tomografia computadorizada de abdome foram normais. Com o diagnóstico de pancreatite aguda foi iniciado o tratamento com hidratação venosa, omeprazol (40 mg/ dia, intravenoso) e octreotida (Sandostatin ${ }^{\circledR}-0,1$ mg subcutâneo, 3 vezes ao dia). Ocorreu melhora rápida dos sintomas e normalização dos níveis de amilase, lipase, glicose e leucócitos.

No primeiro dia da internação foram também observados niveis elevados de triglicerídeos (2089 mg/dL; VN: <200 mg/dL) e de colesterol total $(1.125 \mathrm{mg} / \mathrm{dL} ; \mathrm{VN}:<200 \mathrm{mg} / \mathrm{dL})$. Tinha antecedentes familiares de morte súbita de tios e primos maternos entre os 30 e 40 anos de idade. A mãe era portadora de dislipidemia mista. Foi iniciado o tratamento com dieta para dislipidemia e com bezafibrato $(400 \mathrm{mg} / \mathrm{via}$ oral, duas vezes ao dia). Três dias após, foi associada a cerivastatina $(0,2 \mathrm{mg} / \mathrm{dia})$. Conforme mostrado na Figura 1 , houve uma rápida diminuição dos niveis de triglicerídeos e de colesterol.

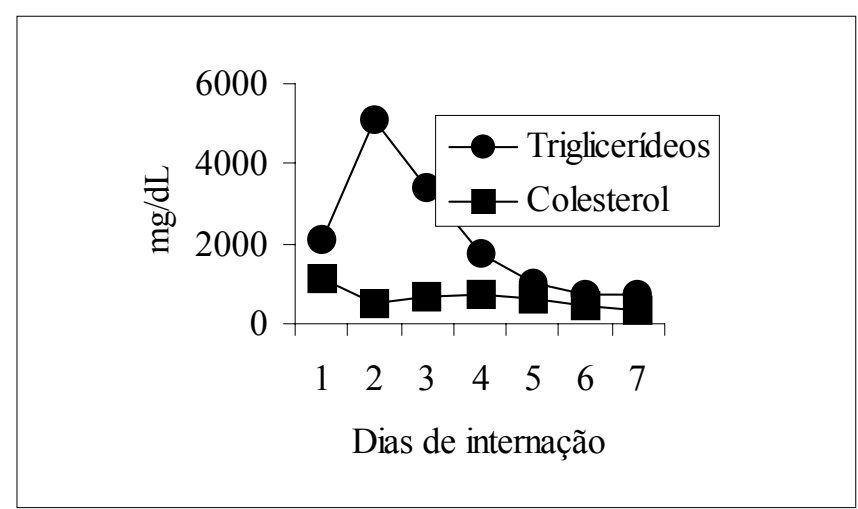

Figura 1 - Rápida diminuição dos níveis de triglicerídeos e colesterol após a instituição de bezafibrato durante a crise de pancreatite aguda, dois meses antes da gravidez.

A paciente recebeu alta hospitalar, sete dias após a internação, com recomendações dietéticas de ingestão diária de 2.000 calorias, das quais $45 \%$ provenientes de carboidratos, 35\% de lipídeos e $20 \%$ de proteinas. Foram mantidas, também, as doses já preconizadas de bezafibrato e cerivastatina. Após dois meses apresentava os seguintes resultados laboratoriais: triglicerídeos $85 \mathrm{mg} / \mathrm{dL}$, colesterol total $169 \mathrm{mg} / \mathrm{dL}$, HDLcolesterol $52 \mathrm{mg} / \mathrm{dL}(\mathrm{VN}$ : $>35 \mathrm{mg} / \mathrm{dL})$, LDLcolesterol $100 \mathrm{mg} / \mathrm{dL}$ (VN: <130 mg/dL), VLDL 17 $\mathrm{mg} / \mathrm{dL}$ (VN: $<40 \mathrm{mg} / \mathrm{dL}$ ) e glicemia $(85 \mathrm{mg} / \mathrm{dL}) . \mathrm{Na}$ ocasião foi suspenso o tratamento com a cerivastatina. Os níveis sangüineos de prolactina, cortisol, TSH, LH e FSH eram normais. Cerca de um mês após a suspensão da cerivastatina, os niveis de triglicerídeos eram de $162 \mathrm{mg} / \mathrm{dL}$ e os de colesterol de $220 \mathrm{mg} / \mathrm{dL}$. A lipoproteína (a) era de $12 \mathrm{mg} / \mathrm{dL}$ (VN: <30 mg/dL), apolipoproteína A de $128 \mathrm{mg} / \mathrm{dL}$ (VN: 100 a $210 \mathrm{mg} / \mathrm{dL}$ ) e apolipoproteína B de 130 (50 a $170 \mathrm{mg} / \mathrm{dL})$. A dose de bezafibrato foi reduzida, então, para $400 \mathrm{mg} /$ dia.

Foi constatado, posteriormente, que nessa ocasião a paciente estava na quarta semana de gestação. Tinha antecedente de uma gestação com evolução e parto sem intercorrências. O bezafibrato foi suspenso na nona semana de gravidez. Conforme mostrado na Figura 2, com a suspensão desse medicamento, os niveis de triglicerídeos aumentaram para $1.148 \mathrm{mg} / \mathrm{dL}$, sendo, então, instituído o uso de ácidos graxos de cadeia ômega-3 (ácidos eicosapentaenóico 14\% e docosahexaenóico $11,13 \%$, preparados em farmácia de manipulação), na dose de $0,5 \mathrm{~g}$, três vezes ao dia, sendo, posteriormente, aumentada para 1,0 $\mathrm{g}$, três vezes ao dia. Ocorreu uma diminuição significativa dos níveis de triglicerídeos, porém ainda se mantiveram elevados até o final da gestação (Figura 2). A concentração sérica de colesterol estava normal no início da gravidez, sofreu um pequeno aumento após a suspensão do bezafibrato e normalizou-se após instituição dos ácidos graxos de cadeia ômega-3 (Figura 2).

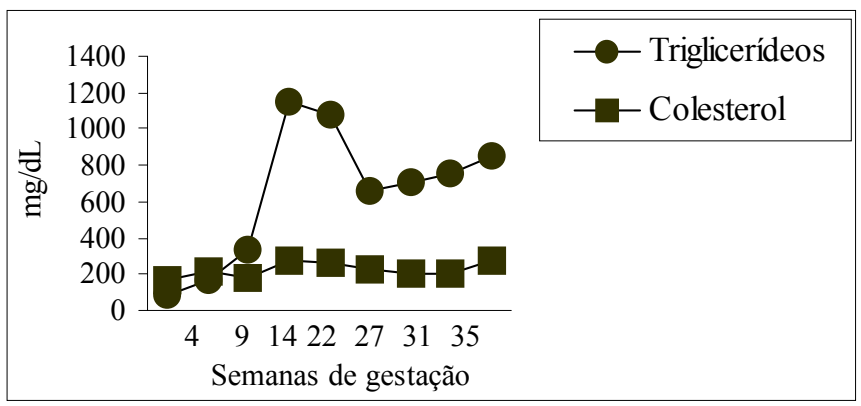

Figura 2 - Comportamento dos níveis de triglicerídeos e colesterol antes, durante e após a gestação. Observa-se um aumento acentuado dos triglicerídeos após a suspensão do bezafibrato ( 9 semanas) e a sua queda com o uso de $1,5 \mathrm{~g}$ (14 semanas) e $3,0 \mathrm{~g}$ (22 semanas) de ácidos graxos de cadeia ômega-3 mantendo-se abaixo de 800 $\mathrm{mg} / \mathrm{dL}$ até após o parto. 
A dieta foi mantida com a mesma estrutura de antes da concepção. O peso da paciente antes da gravidez era de $52 \mathrm{~kg}(\mathrm{IMC}=21,9)$. Evoluiu com ganho ponderal normal, atingindo ao final da gestação $63 \mathrm{~kg}(\mathrm{IMC}=26,6)$. A dopplerfluxometria preditiva para DHEG, realizada com 30 semanas, e a ecocardiografia fetal foram normais.

A paciente teve parto vaginal na $40^{a}$ semana de gestação, com recém-nato saudável, pesando 3.075 g, Apgar 9 e 10 e sem más-formações aparentes. O exame anatomopatológico da placenta evidenciou aumento difuso de nós sinciciais, proeminência das células do citotrofoblasto, fibrina perivilosa, angiomatose e infartos, considerados inespecíficos para hipertrigliceridemia.

Após quatro meses do parto, a paciente encontra-se lactante e, por isso, sendo mantida com 3,0 g de ácidos graxos de cadeia ômega-3. Os níveis sangüineos de triglicerídeos estão em $370 \mathrm{mg} /$ $\mathrm{dL}$ e os de colesterol, em $237 \mathrm{mg} / \mathrm{dL}$.

\section{Discussão}

A paciente aqui descrita é portadora de hipertrigliceridemia familiar, já que apresenta história familiar de dislipidemia e morte súbita em idade precoce. $\mathrm{O}$ fato de esta paciente ter sido acometida de pancreatite aguda, mesmo não-complicada, dois meses antes da fertilização enfatiza a necessidade de se fazer o tratamento da hipertriglicerimia durante a sua gravidez. Como os niveis de triglicerídeos aumentam de 2 a 5 vezes durante a gravide $z^{1}$, era esperado que houvesse uma piora nos seus niveis com a suspensão do bezafibrato. De fato, como mostrado na Figura 2, houve um considerável aumento de triglicerídeos entre a $9^{a}$ e $14^{a}$ semana de gestação. Ainda não foi determinado qual seria o limite nos valores de triglicerídeos na gestação, considerado seguro para evitar complicações devido ao excesso de sua produção. Alguns autores definiram, aleatoriamente, como ponto de corte para se realizar tratamento agressivo em uma paciente com deficiência de lipase lipoproteíca, niveis de triglicerídeos superiores a $2.400 \mathrm{mg} / \mathrm{dL}^{6}$. Nessa mesma paciente foi estabelecido que a sua internação deveria ser mantida até que os niveis dos triglicerídeos estivessem abaixo de $1.500 \mathrm{mg} / \mathrm{dL}^{6}$. Em pacientes portadoras de dislipidemia familiar, outros autores preconizam que os niveis de triglicerideos sejam mantidos entre 855 e $1.280 \mathrm{mg} / \mathrm{dL}^{2}$. A nossa estratégia foi a de mantê-los abaixo de $800 \mathrm{mg} /$ $\mathrm{dL}$, o que corresponderia ao aumento máximo observado durante a gravidez normal ${ }^{1} \mathrm{e}$ aos niveis aceitáveis para o não-desenvolvimento de pancreatite aguda ${ }^{2}$.

Os tratamentos preconizados para diminuir os niveis de triglicerídeos durante a gravidez, em mulheres portadoras de hipertrigliceridemia familiar ou deficiência da lipase lipoproteíca, tem como objetivo prevenir a pancreatite aguda ${ }^{6}$. A utilização de dieta com baixo conteúdo de gordura $(10 \%)$ reduz a produção intestinal de quilomicrons, mas tem o incoveniente de não ser agradável e de pouca aceitação pelas pacientes ${ }^{6}$. Alguns autores utilizam jejum prolongado por seis dias, associado à hidratação com dextrose $5 \%$ em solução de cloreto de sódio a $0,45 \%$, quando os níveis de triglicerídeos ultrapassam $2.400 \mathrm{mg} / \mathrm{dL}^{6}$. Essa conduta é hipocalórica para a gravidez e não pode ser mantida por longo período. Em casos graves, foi utilizada nutrição parenteral total ou plasmaférese de lipoproteinas, mas ambos tratamentos podem ser de grande risco para a paciente ${ }^{4}$. Uma alternativa seria a administração de heparina intravenosa (2.000 a $2.300 \mathrm{U} /$ hora). A heparina intravenosa diminui os niveis de triglicerideos por aumentar a liberação e a atividade da lipase lipoproteíca endotelial, com a segurança de não cruzar a barreira placentária 4 .

Os fibratos são os fármacos preferidos para o tratamento das hipertrigliceridemias ${ }^{7}$. Atuam aumentando a atividade da lipase lipoproteíca, enzima responsável pela hidrólise dos triglicerídeos, além de reduzirem a síntese hepática de VLDL por inibição da síntese e aumento da oxidação dos ácidos graxos ${ }^{7}$. Como observado na Figura 1, o bezafibrato diminuiu dramaticamente os niveis de triglicerídeos durante o episódio de pancreatite aguda e os manteve normais até a ocasião da sua fertilização. No entanto, esses medicamentos são contra-indicados durante a gravidez, bem como as estatinas ${ }^{7}$, que também foram utilizadas pela paciente por um curto período.

Sendo assim, optamos pela substituição do bezafibrato por ácidos graxos de cadeia ômega-3, que têm sido preconizados como opção no tratamento da hipertrigliceridemia ${ }^{7}$. Utilizamos uma dosagem máxima desses ácidos de 3,0 g/dia, considerando o fator diabetogênico próprio da gravidez e a precaução que se deve adotar em relação ao seu uso em pacientes com resistência à insulina ${ }^{7}$. Além disso, a concentração de ácido docosahexaenóico na formulação (466 mg/dia) é um pouco superior às necessidades preconizadas para a gestação $(300 \mathrm{mg} / \mathrm{dia})^{8}$ e ingestão maior de ácidos graxos de cadeia ômega- 3 pode aumentar o risco de sangramentos durante a gravidez ou parto ${ }^{9}$. Essa dose foi utilizada a partir da $22^{\text {a }}$ semana de gestação e foi semelhante àquela utilizada por outros autores $(2,7 \mathrm{~g} / \mathrm{dia})$ em gestantes normais ${ }^{10,11}$. No entanto, a dose média preconizada 
para o tratamento da hipertrigliceridemia, fora da gestação, é de $12 \mathrm{~g} /$ dia e a dose mínima, de 4,5 g/ $\mathrm{dia}^{7}$, superiores àquelas utilizadas nesta paciente. Considerando que, durante a gestação, há um aumento nos niveis de triglicerídeos e, nas portadoras de hipertrigliceridemia familiar e de deficiência da lipase lipoprotéica ocorre uma piora substancial dos seus perfis lipêmicos ${ }^{2}$, a dose relativamente baixa que utilizamos nesta paciente não foi suficiente para normalizar os niveis dos triglicerídeos. Porém, foi adequada para mantê-los em valores inferiores a $800 \mathrm{mg} / \mathrm{dL}$, suficientes para prevenir a pancreatite aguda ${ }^{2}$.

O emprego dos ácidos graxos de cadeia ômega-3, presentes em peixes marinhos, tem sido proposto mesmo em gestações normais, pelos seus efeitos benéficos para a mãe e o feto ${ }^{9}$. Foi sugerido que reduzem a freqüência de doença hipertensiva específica da gestação, tanto por aumentarem a vasodilatação periférica, quanto por diminuírem a agregação plaquetária e a viscosidade sangüínea ${ }^{12}$. Por outro lado, existe uma associação entre niveis séricos baixos de ácidos graxos com aumento no risco de ocorrência de préeclâmpsia ${ }^{13}$. O uso desses ácidos graxos durante o terceiro trimestre pode prolongar a gestação, sem efeitos deletérios no crescimento fetal ou no curso do parto $^{10}$. Em um estudo epidemiológico foi sugerido que, em comunidades nas quais o consumo de peixes e mamíferos marinhos é maior, as mulheres têm niveis mais baixos de pressão arterial sistêmica no final da gravidez e menor incidência de hipertensão arterial específica da gestação ${ }^{14}$.

No entanto, em um estudo aleatório e duplo cego, em que foram utilizados $2,7 \mathrm{~g} /$ dia de ácidos graxos de cadeia ômega-3, não foi demonstrado que estes ácidos tenham efeito protetor em relação à hipertensão arterial, com ou sem proteinúria, ao crescimento intra-uterino e ao parto prematuro ${ }^{15}$. Esse estudo pode apresentar viés, já que as informações sobre a dieta utilizada pelas pacientes somente foram colhidas entre seis meses a três anos após o parto. Além disso, não foram levadas em consideração as diferenças de conteúdo de ácidos graxos de cadeia ômega entre os diversos tipos de peixes.

Em conclusão, o emprego de dieta e de ácidos graxos de cadeia ômega-3 foi efetivo no controle dos niveis de triglicerídeos durante a gestação de uma paciente portadora de dislipidemia familiar. Dados da literatura mostram que o uso dos ácidos graxos de cadeia ômega-3 traz beneficios adicionais, tanto para a evolução normal da gravidez, quanto para o bem-estar fetal.

\section{SUMMARY}

Severe hypertriglyceridemia during pregnancy rarely occurs but it frequently produces complications, such as acute pancreatitis, a serious health risk both for the mother and the fetus. The treatment of a patient who had had acute pancreatitis due to hypertriglyceridemia (triglyceridemia $=$ $5100 \mathrm{mg} / \mathrm{dL}$ ) two months before fecundation is presented in this paper. During gestation, bezafibrate was substituted for $3.0 \mathrm{~g}$ omega-3 fatty acids (14\% eicosapentaenoic and $11.13 \%$ docosahexaenoic acids). With this therapy, the triglyceride levels were maintained below $800 \mathrm{mg} / \mathrm{dL}$, which is considered to be the safe limit to avoid acute pancreatitis. No complication occurred during pregnancy, the patient delivered vaginally (40 weeks), and the newborn (3075 g) did not present any morphological alterations. We conclude that an adequate diet and the use of omega-3 fatty acids were effective in preventing acute pancreatitis in this pregnant woman with serious hypertriglyceridemia. This therapeutic approach may be used alternatively to other treatments currently utilized for familial hypertriglyceridemia in pregnancy.

KEY WORDS: Hypertriglyceridemia. Lipoprotein. Pregnancy: complications. Pancreatitis

\section{Referências}

1. Desoye G, Schweditsch MO, Preiffer KP, Zechner R, Kostner GM. Correlation of hormones with lipid and lipoprotein levels during normal pregnancy and postpartum. J Clin Endocrinol Metab 1987; 64:704-12.

2. Watts GF, Morton K, Jackson P, Lewis B. Management of patients with severe hypertriglyceridaemia during pregnancy: report of two cases with familial lipoprotein lipase deficiency. Br J Obstet Gynaecol 1992; 99:1636.

3. De Chalain TM, Michell WL, Berger GM. Hyperlipidemia, pregnancy and pancreatitis. Surg Gynecol Obstet 1988; 167:469-73.

4. Hsia SH, Connelly PW, Hegele RA. Successful outcome in severe pregnancy-associated hyperlipemia: a case report and literature review. Am J Med Sci 1995; 309:213-8.

5. Montgomery WH, Miller FC. Pancreatitis and pregnancy. Obstet Gynecol 1970; 35:658-64.

6. Sanderson SL, Iverius PH, Wilson DE. Successful hyperlipemic pregnancy. JAMA 1991; 265:185860. 
7. Barcellini A, Martinez TLR. Dislipidemias: diagnóstico e tratamento. In: Ramos OL, Rothschild HA, editores. Atualização Terapêutica. 19 ${ }^{\mathrm{a}}$ ed. São Paulo: Artes Médicas; 1985. p.224-8.

8. Simopoulos AP, Leaf A, Salem N Jr. Workshop on the essentiality of and recommended dietary intakes for omega- 6 and omega-3 fatty acids. J Am Coll Nutr 1999; 18:487-9.

9. Olsen SF. Consumption of marine n-3 fatty acids during pregnancy as a possible determinant of birth weight. Epidemiol Rev 1993; 15:399-413.

10.Olsen SF, Sorensen JD, Secher NJ, et al. Randomised controlled trial of effect of fish-oil supplementation on pregnancy duration. Lancet 1992; 339:1003-7.

11. Onwude JL, Lilford RJ, Hjartardottir H, Staines A, Tuffnell D. A randomised double blind placebo controlled trial of fish oil in high risk pregnancy. Br J Obstet Gynaecol 1995; 102:95-100.

12.Secher NJ, Olsen SF. Fish-oil and pre-eclampsia. Br J Obstet Gynaecol 1990; 97:1077-9.
13. Williams MA, Zingheim RW, King IB, Zebelman AM. Omega-3 fatty acids in maternal erythrocytes and risk of preeclampsia. Epidemiology 1995; 6:2327 .

14. Gerrard J, Popeski D, Ebbeling L, Brown P, Hornstra G. Dietary omega 3 fatty acids and gestational hypertension in the Inuit. Arctic Med Res 1991; 50 Suppl:763-7.

15. Kesmodel U, Olsen SF, Salvig JD. Marine n-3 fatty acid and calcium intake in relation to pregnancy induced hypertension, intrauterine growth retardation, and preterm delivery. A case-control study. Acta Obstet Gynecol Scand 1997; 76:3844.

\section{Agradecimento:}

A Luiz Gustavo Domingues Casulari da Motta pela ajuda na confecção deste texto.

\section{COMUNICADO AOS ASSOCIADOS E LEITORES}

\section{Para informações e consultas temos à disposição quatro endereços eletrônicos:}

\section{febrasgopresiden@uol.com.br} secretaria_executiva@febrasgo.org.br publicacoes@febrasgo.org.br tego_habilitacao@febrasgo.org.br 